DFF 452/02/2010

\title{
Algebraic arctic curves in the domain-wall six-vertex model
}

\author{
F. Colomo, V. Noferini, and A. G. Pronko
}

\begin{abstract}
The arctic curve, i.e. the spatial curve separating ordered (or 'frozen') and disordered (or 'temperate) regions, of the six-vertex model with domain wall boundary conditions is discussed for the rootof-unity vertex weights. In these cases the curve is described by algebraic equations which can be worked out explicitly from the parametric solution for this curve. Some interesting examples are discussed in detail. The upper bound on the maximal degree of the equation in a generic root-of-unity case is obtained.
\end{abstract}

\section{Introduction}

It is commonly known that in strongly correlated systems boundary conditions may lead to emergence of spatial separation of phases, e.g., order and disorder. Famous examples of such systems are domino tiling of large Aztec diamonds [1, 2] and lozenge tilings of large hexagon [3, 4]. Another example, more elaborated and with important combinatorial applications as particular cases, is represented by the six-vertex model with domain wall boundary conditions [5 14].

Recently, for this model a progress was achieved in finding an explicit form of the curve separating the disordered and ferroelectrically ordered phases (called Arctic curve, by analogy with Arctic circle of domino tilings). In [15] a conjectural expression for the Arctic curve was derived for various particular cases of Boltzmann weights, with one of them providing the limit shape of large alternating-sign matrices. In [16] this result for the Arctic curve was extended to the case of generic Boltzmann weights of the six-vertex model corresponding to its disordered regime. The case of anti-ferroelectric regime was considered in [17].

In this paper we further discuss the Arctic curve of the domain-wall six-vertex model, focussing on the special cases of Boltzmann weights in the disordered regime, where the Arctic curve, generally transcendental, becomes algebraic. The six-vertex model in these cases is sometimes referred to as the six-vertex model at 'roots of unity' (the term comes mostly from the quantum-group context). Some of these cases were already considered in [16]. Here we explain in detail how the algebraic equation can be derived from the general parametric solution for the curve, when the Boltzmann weights corresponds to the 'root-of-unity' cases. In the specific case of dimer 
models on planar bipartite graphs, the relation between Arctic curves and algebraic curves was investigated in [18 20].

The outline of the paper is as follows. In the next section we recall the result for the Arctic curve of the domain-wall six-vertex model in its disordered regime. The specificities of the 'root-of-unity' cases are explained in section 3. Some interesting particular cases are exposed in section 4. We end up by discussing the upper limit bound for the degree of the algebraic equation for the Arctic curve for generic 'root-of-unity' case in section 5 . Finally, some technical aspects are discussed in the appendix.

\section{Arctic curve of the disordered regime}

We start with recalling the result of paper [16] on the arctic curve of the six-vertex model with domain wall boundary conditions in its disordered regime. We first recall how the Arctic curve arises in the model and next explain the result.

We consider the six-vertex model on a square lattice formed by intersection of equal number of vertical and horizontal lines, with all states on the boundaries fixed in the special way, called the domain wall boundary conditions [5]. Using the arrow language of states on edges, these boundary conditions mean that all arrows on top and bottom boundaries are incoming, while on the left and right boundaries they are outgoing.

The Boltzmann weights, usually denoted as $a, b$ and $c$, are parameterized as

$$
a=\sin (\lambda+\eta), \quad b=\sin (\lambda-\eta), \quad c=\sin 2 \eta,
$$

where $\lambda$ and $\eta$ are the 'rapidity' variable and the 'crossing' parameter, respectively. The disordered regime corresponds to $\lambda$ and $\eta$ real and satisfying

$$
\eta<\lambda<\pi-\eta, \quad 0<\eta<\pi / 2 .
$$

It is useful to recall that physical regimes of the six-vertex model are characterized by the parameter $\Delta=\left(a^{2}+b^{2}-c^{2}\right) / 2 a b$, and the disordered regime corresponds to $|\Delta|<1$; in our parametrization we have $\Delta=\cos 2 \eta$.

The Arctic curve describes spatial separation of phases, of ferroelectric order and disorder. The effect of separation of phases is related to the fact that in the domain-wall six-vertex model ordered configurations on the boundary can induce, through the ice-rule, a macroscopic order inside the lattice.

The notion of phase separation, and hence the Arctic curve, acquires a precise meaning in the scaling limit, i.e., when the number of lines of the lattice (in each direction) tends to infinity and the lattice spacing vanishes, while the total size of the system (in each direction) is kept fixed. For the domain-wall six-vertex model one can assume that the lattice is scaled to the square $[0,1] \times[0,1]$. To describe points of this square we use coordinates $(x, y)$ with $x, y \in[0,1]$. As in [16], we consider the coordinate system in which the $y$-axis is reversed, with the origin at the top left corner of the square. 
The phase separation of domain-wall six-vertex model in its disordered regime is characterized by appearance of five regions in the scaling limit: four regions of ferroelectric order, $F_{\mathrm{NW}}, \mathrm{F}_{\mathrm{NE}}, \mathrm{F}_{\mathrm{SE}}$, and $\mathrm{F}_{\mathrm{SW}}$ in the four corners of the square, and one region of disorder $\mathrm{D}$, in the centre. The region of disorder is sharply delimited by a curve $\mathcal{A}$, called the Arctic curve. The Arctic curve and the square have four contact points, located each one on a side of the square. The arctic curve in the disordered regime consists of four portions,

$$
\mathcal{A}=\Gamma_{\mathrm{NW}} \cup \Gamma_{\mathrm{NE}} \cup \Gamma_{\mathrm{SE}} \cup \Gamma_{\mathrm{SW}},
$$

where $\Gamma_{i}$ separates the region $\mathrm{F}_{i}(i=\mathrm{NW}, \mathrm{NE}, \mathrm{SE}, \mathrm{SW})$ from the internal region of disorder D. Due to the symmetries of the model (e.g., crossing symmetry) given, for example, portion $\Gamma_{\mathrm{NW}}$, one can easily obtain the remaining three portions $\Gamma_{\mathrm{NE}}, \Gamma_{\mathrm{SE}}$, and $\Gamma_{\mathrm{SW}}$ (for details, see [16], section 2) and hence obtain the whole curve $\mathcal{A}$.

To describe the curve $\Gamma_{\mathrm{NW}}$, one can introduce a function $\Upsilon(x, y ; \lambda)$ where $x$ and $y$ are coordinates in the scaling limit and $\lambda$ is the parameter of the weights,

$$
\Gamma_{\mathrm{NW}}: \Upsilon(x, y ; \lambda)=0, \quad x, y \in[0, \kappa] .
$$

Due to symmetries of the model, this function obeys $\Upsilon(x, y ; \lambda)=\Upsilon(y, x ; \lambda)$. The quantity $\kappa=\kappa(\lambda)$ gives location of the contact points of the Arctic curve, in particularly, the points $(\kappa, 0)$ and $(0, \kappa)$ are the end-points of the curve $\Gamma_{\mathrm{NW}}$. Explicitly (see [16], section 3 ), it reads

$$
\kappa=\frac{\alpha \cot \alpha(\lambda-\eta)-\cot (\lambda+\eta)}{\cot (\lambda-\eta)-\cot (\lambda+\eta)},
$$

where

$$
\alpha=\frac{\pi}{\pi-2 \eta} .
$$

In the case of $\lambda=\pi / 2$, i.e., when the weights $a$ and $b$ are equal, see (2.1), one has $\kappa=1 / 2$ for all values of the parameter $\eta$.

The explicit form of the function $\Upsilon(x, y ; \lambda)$ is significantly determined by the value of $\eta$. Furthermore, it turns out that function $\Upsilon(x, y ; \lambda)$ for generic values of $\eta$ is a non-algebraic, or transcendental, function. This follows from the parametric solution for the curve $\Gamma_{\mathrm{NW}}$, obtained in [16].

Namely, let $\zeta$ be real and taking values in the interval $[0, \pi-\lambda-\eta]$. Let us consider the function $f(x, y ; \lambda ; \zeta)$ which depends on $x$ and $y$ linearly, and is given explicitly by the formula

$$
\begin{array}{r}
f(x, y ; \lambda ; \zeta)=\frac{x \sin 2 \eta}{\sin (\zeta+\lambda-\eta) \sin (\zeta+\lambda+\eta)}+\frac{y \sin 2 \eta}{\sin \zeta \sin (\zeta+2 \eta)} \\
-\frac{\sin (\lambda+\eta)}{\sin \zeta \sin (\zeta+\lambda+\eta)}+\frac{\alpha \sin \alpha(\lambda-\eta)}{\sin \alpha \zeta \sin \alpha(\zeta+\lambda-\eta)} .
\end{array}
$$

The parameter $\zeta$ parameterizes the curve $\Gamma_{\mathrm{NW}}$ as it runs over the interval $[0, \pi-\lambda-\eta]$ while the curve is given in the parametric form

$$
x=X(\zeta), \quad y=Y(\zeta) .
$$


Writing simply $f(\zeta)$ for function $f(x, y ; \lambda ; \zeta)$, the functions $X(\zeta)$ and $Y(\zeta)$ correspond to the solution, for unknowns $x$ and $y$, of the linear system of equations

$$
f(\zeta)=0, \quad f^{\prime}(\zeta)=0,
$$

where the prime denotes derivative. For later use, we mention that equations (2.9) are equivalent to the condition that function $f(\zeta)$ must have a double root; each point of the curve $\Gamma_{\mathrm{NW}}$ corresponds to a real value of this double root, in the interval $[0, \pi-\lambda-\eta]$.

Functions $X(\zeta)$ and $Y(\zeta)$ are related to each other as $X(\zeta)=Y(\pi-\lambda-$ $\eta-\zeta)$ that follows from the obvious property

$$
f(x, y ; \lambda ; \zeta)=f(y, x ; \lambda ; \pi-\lambda-\eta-\zeta),
$$

reflecting the $x \leftrightarrow y$ symmetry of the curve $\Gamma_{\mathrm{NW}}$. Explicit expressions for functions $X(\zeta)$ and $Y(\zeta)$ can be found in [16], see equations (6.16)-(6.19) therein; for our discussion below expression (2.7) and equations (2.9) are sufficient.

\section{3. 'Root-of-unity' cases}

Let us now focus on the case when the parameter $\eta$ is such that the parameter $\alpha$ is a rational number

$$
\alpha=\frac{n}{d} \quad(d<n),
$$

where $n$ and $d$ are some co-prime integers. The parameter $\eta$ reads

$$
\eta=\frac{\pi}{2} \cdot \frac{n-d}{n}
$$

and it corresponds to the so-called six-vertex model at a 'root of unity' (the root of unity here is the deformation parameter $q$ of the underlying quantum group, $q=\exp 2 \mathrm{i} \eta=-\exp (-\mathrm{i} \pi / \alpha))$.

To make the subsequent discussion simpler, we start with rewriting function (2.7) in a more symmetric form. Namely, we introduce parameters $\varphi$ and $\varkappa$ related to parameters $\zeta$ and $\lambda$ by a linear change of variables

$$
\varphi=\zeta-\varkappa, \quad \varkappa=\frac{\pi-\lambda-\eta}{2} .
$$

Introducing function $g(x, y ; \varkappa ; \varphi):=f(x, y ; \lambda ; \zeta)$, which we shall write simply as $g(\varphi)$, we arrive at a more symmetric expression

$$
\begin{array}{r}
g(\varphi)=\frac{x \sin 2 \eta}{\sin (\varkappa-\varphi) \sin (\varkappa+2 \eta-\varphi)}+\frac{y \sin 2 \eta}{\sin (\varkappa+\varphi) \sin (\varkappa+2 \eta+\varphi)} \\
-\frac{\sin 2 \varkappa}{\sin (\varkappa+\varphi) \sin (\varkappa-\varphi)}+\frac{\alpha \sin 2 \alpha \varkappa}{\sin \alpha(\varkappa+\varphi) \sin \alpha(\varkappa-\varphi)} .
\end{array}
$$

Note that new parameters run over the values $\varphi \in[-\varkappa, \varkappa]$ and $\varkappa \in(0, \pi / 2 \alpha)$, and $\alpha(1<\alpha<\infty)$ in (3.4) is still arbitrary. Evidently, equations (2.9) now 
read $g(\varphi)=0$ and $g^{\prime}(\varphi)=0$ and we have simply reformulated the parametric expression for the curve $\Gamma_{\mathrm{NW}}$ just by shifting the parameter of the curve.

Using the well-known identity

$$
\sin n \varphi=2^{n-1} \prod_{j=0}^{n-1} \sin \left(\varphi+\frac{\pi j}{n}\right)
$$

where $n$ is an arbitrary positive integer, one can easily derive the identity

$$
\sin \frac{n}{d}(\varkappa \pm \varphi)=\left(\sin \frac{\varphi}{d}\right)^{n} \sin \frac{n}{d} \varkappa \prod_{j=0}^{n-1}\left(\cot \frac{\varphi}{d} \pm \cot \left(\frac{\varkappa}{d}+\frac{\pi j}{n}\right)\right) .
$$

Formally setting here $n=d$, we also have the identity

$$
\sin (\varkappa \pm \varphi)=\left(\sin \frac{\varphi}{d}\right)^{d} \sin \varkappa \prod_{k=0}^{d-1}\left(\cot \frac{\varphi}{d} \pm \cot \left(\frac{\varkappa}{d}+\frac{\pi j}{d}\right)\right) .
$$

Let us now rewrite the function $g(\varphi)$ for the 'root-of-unity' cases using relations (3.6) and (3.7). Denoting

$$
t=\cot \frac{\varphi}{d}
$$

and taking into account that $\sin ^{2}(\varphi / d)=\left(t^{2}+1\right)^{-1}$, we arrive at the following formula:

$$
\begin{aligned}
g(\varphi) & =x \rho \frac{\left(t^{2}+1\right)^{d}}{\prod_{k}\left(t-v_{k}\right)\left(t-u_{k}\right)}+y \rho \frac{\left(t^{2}+1\right)^{d}}{\prod_{k}\left(t+v_{k}\right)\left(t+u_{k}\right)} \\
& -2 \cot \varkappa \frac{\left(t^{2}+1\right)^{d}}{\prod_{k}\left(t-v_{k}\right)\left(t+v_{k}\right)}+2 \alpha \cot \alpha \varkappa \frac{\left(t^{2}+1\right)^{n}}{\prod_{j}\left(t-w_{j}\right)\left(t+w_{j}\right)} .
\end{aligned}
$$

Here

$$
\rho=\frac{\sin 2 \eta}{\sin \varkappa \sin (\varkappa+2 \eta)}
$$

and the numbers $v_{k}, u_{k}(k=0, \ldots, d-1)$ and $w_{j}(j=0, \ldots, n-1)$ are

$$
\begin{aligned}
& v_{k}=\cot \left(\frac{\varkappa}{d}+\frac{\pi k}{d}\right), \\
& u_{k}=\cot \left(\frac{\varkappa}{d}+\frac{2 \eta}{d}+\frac{\pi k}{d}\right), \\
& w_{j}=\cot \left(\frac{\varkappa}{d}+\frac{\pi j}{n}\right) .
\end{aligned}
$$

The products in (3.9) are taken over the indicated values of the integers $j$ or $k$. 
Clearly, from formula (3.9) it follows that function $g(\varphi)$ is a rational function of the variable $t$, with the following structure

$$
g(\varphi)=\frac{\left(t^{2}+1\right)^{d}}{Q(t)} P(t) .
$$

Here $P(t)$ and $Q(t)$ are polynomials: $Q(t)$ is the common denominator of the four terms in (3.9) while $P(t)$ is the resulting numerator. Evidently, the condition that function $g(\varphi)$ has a double real root in the interval $\varphi \in$ $[-\varkappa, \varkappa]$, which provides a parametric form for the curve $\Gamma_{\mathrm{NW}}$, now translates into the condition that polynomial $P(t)$ has a double real root in the interval $t \in\left[-\infty,-v_{0}\right] \cup\left[v_{0}, \infty\right]$.

It is well known a polynomial has a double root (not necessarily real) if and only if its discriminant is equal to zero. The discriminant, in turn, is a homogenous polynomial in the coefficients of the polynomial. The coefficients of $P(t)$ are linear functions of $x$ and $y$, and therefore requiring the discriminant of $P(t)$ to be equal to zero provides an equation for an algebraic curve. The portion of such algebraic curve corresponding to the additional requirement that the double root must lie in the real interval $t \in\left[-\infty,-v_{0}\right] \cup\left[v_{0}, \infty\right]$ is the portion $\Gamma_{\mathrm{NW}}$ of the Arctic curve, lying in the region $x, y \in[0, \kappa]$ of the unit square.

To be more specific, let $P(t)$ be a polynomial of degree $m(m \geqslant 2)$ with non-vanishing leading coefficient, $p_{m} \neq 0$. The discriminant of $P(t)$ can be written as

$$
D_{m}(P)=(-1)^{m(m-1) / 2} \operatorname{det} S_{m-1, m-1}\left(\tilde{P}, P^{\prime}\right) .
$$

where $S_{m-1, m-1}\left(\tilde{P}, P^{\prime}\right)$ is the Sylvester matrix of the two polynomials $\tilde{P}(t)=$ $m P(t)-t P^{\prime}(t)$ and $P^{\prime}(t)$, where the prime denotes derivative. Further details are given in the appendix. The condition that $P(t)$ has a double root is just

$$
D_{m}(P)=0 \text {. }
$$

In view of the above considerations, we conclude that in the 'root-of-unity' cases this equation contains equation (2.4), with $D_{m}(P)$ providing (modulo the problem of reducibility) an explicit expression for function $\Upsilon(x, y ; \lambda)$.

In the next section we consider several interesting examples of the 'rootof-unity' cases, namely, we consider the cases of $\alpha=2,3,3 / 2,4,5 / 2$. The case of $\alpha=2$ is the free-fermion point and the Arctic curve is the Arctic Ellipse (or Arctic Circle, at $\lambda=\pi / 2$ ), see [15, 21] and references therein. The cases $\alpha=3,3 / 2$, at $\lambda=\pi / 2$, were already treated in papers [15, 16]; here we discuss the case of generic $\lambda$. The cases of $\alpha=4,5 / 2$ are considered for the first time.

In particular, we find that the degree of $P(t)$ is significantly determined by the integers $n$ and $d$ defining the parameter $\alpha=n / d$. We also find that at $\lambda=\pi / 2$ a simplification in the degree may occur. Motivated by the examples considered below, we discuss the upper bounds on the degree of algebraic equations for the Arctic curve in section 5. 


\section{Examples}

4.1. Case $\alpha=2(\eta=\pi / 4$ or $\Delta=0)$. In this case the numbers $v_{k}$, $u_{k}(k=0, \ldots, d-1)$ and $w_{j}(j=0, \ldots, n-1)$, see (3.11), which describe location of poles are $(d=1$ and $n=2)$ :

$$
v_{0}=\cot \varkappa, \quad u_{0}=-\tan \varkappa, \quad w_{0}=\cot \varkappa, \quad w_{1}=-\tan \varkappa .
$$

Since $v_{0}=w_{0}$ and $u_{0}=w_{1}$, the common denominator is given by the denominator of the last term in (3.4), and hence the polynomial $P(t)$ is quadratic. Computing, we obtain

$$
P(t)=\frac{2}{\sin 2 \varkappa}\left[(x+y-1+\cos 2 \varkappa) t^{2}+2 \cot 2 \varkappa \cdot t-(x+y-1-\cos 2 \varkappa)\right] .
$$

Evaluating the discriminant of $P(t)$ and cancelling an overall factor, for the Arctic curve we find

$$
\frac{(x+y-1)^{2}}{\cos ^{2} 2 \varkappa}+\frac{(x-y)^{2}}{\sin ^{2} 2 \varkappa}-1=0 .
$$

This is of course the well known result of the Arctic Ellipse (see, e.g., [21] and references therein).

4.2. Case $\alpha=3(\eta=\pi / 3$ or $\Delta=-1 / 2)$. In this case the denominators in (3.9) are governed by numbers $v_{0}, u_{0}, w_{0}, w_{1}$, and $w_{2}$, and we have relations

$$
v_{0}=w_{0}, \quad u_{0}=w_{2} .
$$

Thus the common denominator is given by the denominator of the last term in (3.9) and in this case $P(t)$ is a polynomial of degree 4. After some algebra we find

$$
\begin{aligned}
P(t)= & \frac{2}{\sin ^{2} 3 \nu}\left\{\sin 3 \nu[\sin \nu \cdot \Lambda-\cos \nu(1-4 \cos 2 \nu)] t^{4}\right. \\
& +4 \sin ^{2} \nu \sin 2 \nu \cdot \Theta t^{3}-[3 \cos 2 \nu \cdot \Lambda+\sin 2 \nu(1-4 \cos 4 \nu)] t^{2} \\
& \left.-4 \cos ^{2} \nu \sin 2 \nu \cdot \Theta t+\cos 3 \nu[\cos \nu \cdot \Lambda+\sin \nu(1+4 \cos 2 \nu)]\right\}
\end{aligned}
$$

where

$$
\Lambda=\sqrt{3}(1-x-y), \quad \Theta=\sqrt{3}(x-y), \quad \nu=\varkappa+\frac{\pi}{3} .
$$

Evaluating the discriminant of this polynomial and equating it to zero gives the equation for Arctic curve. In this case it is of degree 6. The explicit expression for generic $\lambda$ (or $\varkappa$ ) is too cumbersome to be given here (though it is just of degree 6 , each coefficient is a complicated function of $\varkappa$ ).

In the special case of $\lambda=\pi / 2$, that corresponds to the choice $\varkappa=\pi / 12$, the expression for the Arctic curve simplifies considerably and one arrives 
at equation

$$
\begin{gathered}
324\left(x^{6}+y^{6}\right)+1620\left(x^{5} y+x y^{5}\right)+3429\left(x^{4} y^{2}+x^{2} y^{4}\right)+4254 x^{3} y^{3} \\
-972\left(x^{5}+y^{5}\right)-1458\left(x^{4} y+x y^{4}\right)-2970\left(x^{3} y^{2}+x^{2} y^{3}\right)-6147\left(x^{4}+y^{4}\right) \\
-9150\left(x^{3} y+x y^{3}\right)-17462 x^{2} y^{2}+13914\left(x^{3}+y^{3}\right)+24086\left(x^{2} y+x y^{2}\right) \\
-11511\left(x^{2}+y^{2}\right)-17258 x y+4392(x+y)-648=0 .
\end{gathered}
$$

This formula describes the limit shape of 3-enumerated alternating-sign matrices and it was obtained for the first time in [15].

4.3. Case $\alpha=3 / 2(\eta=\pi / 6$ or $\Delta=1 / 2)$. In this case, with $\lambda$ (or $\varkappa$ ) is generic, the polynomial $P(t)$ is of degree 6 . Indeed, for $d=2$ and $n=3$ the denominators in (3.9) are controlled by seven numbers $v_{0}, v_{1}, u_{0}, u_{1}$, $w_{0}, w_{1}$, and $w_{2}$ among those there are two pairs of coinciding ones,

$$
v_{0}=w_{0}, \quad u_{1}=w_{2} .
$$

Already the resulting polynomial $P(t)$ is too lengthy in its coefficients to be presented here, not even to mention its discriminant, giving the Arctic curve, which is of degree 10 in this case.

This curve is however interesting in the fact that it factorizes when one specializes to the case of $\lambda=\pi / 2$. Namely, an expression of degree 10 factorizes into several factors among which only one factor, of degree 2 , corresponds to the Arctic curve, while the remaining factors are nonvanishing for the allowed values of $x$ and $y$.

The origin of such factorization becomes evident if one comes back to formula (3.9). Indeed, at $\lambda=\pi / 2$, which corresponds to $\varkappa=\pi / 6$, we have two more additional relations

$$
v_{1}=-w_{1}, \quad u_{0}=-w_{2} .
$$

Correspondingly, in this special case the polynomial $P(t)$ is quadratic, and it reads

$$
P(t)=\sqrt{3}(x+y-2+\sqrt{3}) t^{2}+6(x-y) t-\sqrt{3}(x+y-2-\sqrt{3}) .
$$

Evaluating the discriminant, and setting it equal to zero, we obtain equation

$$
(x+y-2)^{2}+3(x-y)^{2}-3=0 .
$$

Evidently, this equation describes an ellipse inscribed into square $[0,2] \times$ $[0,2]$; we recall that the Arctic curve is given only by a portion of this ellipse, for which $x, y \in[0,1 / 2]$.

Equation (4.11) is an important example of Arctic curve, since it describes the limit shape of large alternating-sign matrices (within 1-enumeration scheme). Equation (4.11) was obtained previously in papers [15, 16]. Interestingly, expanding terms in (4.11) we obtain

$$
4 x^{2}-4 x y+4 y^{2}-4(x+y)+1=0,
$$


which differs just in a single term, namely $-4 x y$, from equation (4.3) at $\lambda=\pi / 2$ (corresponding to $\varkappa=\pi / 8$ in that case).

4.4. Case $\alpha=4(\eta=3 \pi / 8$ or $\Delta=-\sqrt{2} / 2)$. In this case the denominators in (3.9) are controlled by the numbers $v_{0}, u_{0}$ and $w_{0}, \ldots, w_{3}$. Similarly to the cases of $\alpha=2$ and $\alpha=3$, the common denominator is given by denominator of the last term in (3.9), due to the relations $v_{0}=w_{0}$ and $u_{0}=w_{3}$. Now the polynomial $P(t)$ has degree 6 , and the Arctic curve is given by equation of degree 10 . At $\lambda=\pi / 2$ no simplification of the degree of $P(t)$ is possible, and the Arctic curve remains of degree 10; the explicit expression for the equation, which contains irrational coefficients involving $\sqrt{2}$, is lengthy and uninformative.

The case of $\alpha=4$ has been included in the list of our examples to show some common properties of the cases of integer values of $\alpha$, namely, that the common denominator is given by the denominator of the last term in (3.9), and that no simplification of the degree occurs at $\lambda=\pi / 2$. For these reasons considering the integer values of $\alpha$ is in fact not illuminating; the cases $\alpha=2$ and $\alpha=3$ are somewhat exceptional, in view of their well-known relations to important enumeration problems in combinatorics.

4.5. Case $\alpha=5 / 2(\eta=3 \pi / 10$ or $\Delta=(1-\sqrt{5}) / 4)$. In this case, for generic values of $\lambda$, the polynomial $P(t)$ is of degree 10 and, correspondingly, the Arctic curve is given by equation of degree 18. We restrict ourselves henceforth to the case of $\lambda=\pi / 2$ where the degree of polynomial $P(t)$ is just 6 . Indeed, for $\lambda=\pi / 2$ we have in this case $\varkappa=\pi / 10$ and for the numbers $v_{0}, v_{1}, u_{0}, u_{1}$ and $w_{0}, \ldots, w_{4}$ determining the denominators of terms in (3.9) we have relations

$$
v_{0}=w_{0}, \quad u_{1}=w_{4}, \quad v_{1}=-w_{2}, \quad u_{0}=-w_{3} .
$$

Due to these relations the common denominator $Q(t)$ in this case is given by denominator of the last term in (3.9). Computing the polynomial $P(t)$ we obtain the expression

$$
\begin{aligned}
P(t)=\rho & \left\{\left(x+y-\frac{1+\sqrt{5}}{2}+\sqrt{5} \sigma\right) t^{6}+8 \sigma(x-y) t^{5}\right. \\
& -\left((3+4 \sqrt{5})(x+y)+\frac{17-15 \sqrt{5}}{2}-3 \sqrt{5} \sigma\right) t^{4}-16 \sigma(x-y) t^{3} \\
& +\left((3+4 \sqrt{5})(x+y)+\frac{17-15 \sqrt{5}}{2}+3 \sqrt{5} \sigma\right) t^{2}+8 \sigma(x-y) t \\
& \left.-\left(x+y-\frac{1+\sqrt{5}}{2}-\sqrt{5} \sigma\right)\right\}
\end{aligned}
$$

where $\rho=\sqrt{2(5+\sqrt{5})}$ and $\sigma=\sqrt{\frac{5-\sqrt{5}}{8}}=\sqrt{5} \rho^{-1}$.

The discriminant of polynomial (4.14), which is of degree 10 in $x$ and $y$, appears to factorize into two polynomials, of degrees 2 and 8 . In other words, 
the corresponding algebraic curve has two components. The quadratic factor is

$$
\begin{aligned}
2 x^{2}-(1-\sqrt{5}) x y & +2 y^{2}-2 x-2 y+3-\sqrt{5} \\
& =\frac{3+\sqrt{5}}{4}(x+y-3+\sqrt{5})^{2}+\frac{5-\sqrt{5}}{4}(x-y)^{2}
\end{aligned}
$$

where the second line shows that it is positive for all values of $x$ and $y$, except point $x=y=(3-\sqrt{5}) / 2$ where it vanishes. It is easily verified that this component (consisting in just one point) corresponds to the double root $t=i$ (or $t=-i$ ) of $P(t)$. Hence, the Arctic curve is given by the other component, i.e. by the factor degree of 8 . The equation for the Arctic curve reads:

$$
\begin{aligned}
& 128\left(x^{8}+y^{8}\right)-512(1-\sqrt{5})\left(x^{7} y+x y^{7}\right)+1536\left(x^{6} y^{2}+x^{2} y^{6}\right) \\
& +512(25-9 \sqrt{5})\left(x^{5} y^{3}+x^{3} y^{5}\right)+256(83-32 \sqrt{5}) x^{4} y^{4}-512\left(x^{7}+y^{7}\right) \\
& +512(13-8 \sqrt{5})\left(x^{6} y+x y^{6}\right)-512(65-24 \sqrt{5})\left(x^{5} y^{2}+x^{2} y^{5}\right) \\
& -512(267-112 \sqrt{5})\left(x^{4} y^{3}+x^{3} y^{4}\right)-96(74-29 \sqrt{5})\left(x^{6}+y^{6}\right) \\
& +64(435-182 \sqrt{5})\left(x^{5} y+x y^{5}\right)-32(2874-1313 \sqrt{5})\left(x^{4} y^{2}+x^{2} y^{4}\right) \\
& +128(7233-3214 \sqrt{5}) x^{3} y^{3}+32(722-261 \sqrt{5})\left(x^{5}+y^{5}\right) \\
& +32(6132-2663 \sqrt{5})\left(x^{4} y+x y^{4}\right)-64(6911-3154 \sqrt{5})\left(x^{3} y^{2}+x^{2} y^{3}\right) \\
& -4(12403-5205 \sqrt{5})\left(x^{4}+y^{4}\right)-4(97677-42727 \sqrt{5})\left(x^{3} y+x y^{3}\right) \\
& \quad-16(18381-8009 \sqrt{5}) x^{2} y^{2}+360(167-77 \sqrt{5})\left(x^{3}+y^{3}\right) \\
& +16(58205-26052 \sqrt{5})\left(x^{2} y+x y^{2}\right)-(31813-15751 \sqrt{5})\left(x^{2}+y^{2}\right) \\
& -2(385088-173179 \sqrt{5}) x y+(5689-3283 \sqrt{5})(x+y)+85 \sqrt{5}+87=0 .
\end{aligned}
$$

Even though the resulting equation is not illuminating, the whole example of the case of $\alpha=5 / 2$ appears very instructive. Indeed, we observe again, like in the case of $\alpha=3 / 2$, a reduction in the degree of polynomial $P(t)$ at $\lambda=\pi / 2$. Furthermore, we observe that the Arctic curve can be of a lower degree, with respect to the degree expected from the one of $P(t)$, due to additional factorization.

As we show below, the drop down of degrees of polynomials $P(t)$ is a common feature of all cases with half-integer values of $\alpha$ at $\lambda=\pi / 2$. On the other hand the additional factorization of its discriminant cannot be tackled by simple means. It corresponds to the existence of a family of complex double roots for $P(t)$, and hence to the existence of an additional component in the algebraic curve defined by the condition of vanishing resultant for $P(t)$. The identification and elimination of such additional component can be performed, at least in principle, in each specific case, but no general procedure is available. 


\section{Upper bounds for degrees of the curves}

In the examples considered above it is evident that the degree of polynomial $P(t)$ and hence the degree for the Arctic curve as equation on $x$ and $y$, essentially depends on the values of $d$ and $n$, the co-prime integers determining value of the parameter $\alpha$.

Using formulae of section 3 it is fairly easy to find an expression for the common denominator in (3.12). In this way one can infer general properties of the numerator and hence find the degree of $P(t)$.

Assuming that we are in the case of generic $\lambda$ (or $\varkappa$ ) we have relations

$$
v_{0}=w_{0}, \quad u_{d-1}=w_{n-1} .
$$

Indeed, the first relation is immediate, while the second follows from $\pi$ periodicity of cotangent function and formula

$$
\frac{2 \eta}{d}=\frac{\pi}{d}-\frac{\pi}{n}
$$

Since $\varkappa$ is assumed to be arbitrary, no further relations among numbers $v_{0}, \ldots, v_{d-1}, u_{0}, \ldots, u_{d-1}$, and $w_{0}, \ldots, w_{n-1}$ are possible.

Having relations (5.1) we obtain that the common denominator $Q(t)$ in formula (3.12) reads

$$
Q(t)=\prod_{j=0}^{n-1}\left(t-w_{j}\right)\left(t+w_{j}\right) \prod_{k=1}^{d-1}\left(t-v_{k}\right)\left(t+v_{k}\right) \prod_{k=0}^{d-2}\left(t-u_{k}\right)\left(t+u_{k}\right) .
$$

Using this expression one can easily see that each of the four terms in (3.9) contributes to the numerator with the terms of the same degree in $t$. The result can be summarised as follows.

Proposition 1. In the case of $\alpha=n / d$ where $n$ and $d$ are co-prime integers and $\lambda$ is generic, the polynomial $P(t)$ in (3.12) has degree less than or equal to $2 d+2 n-4$.

Proof. A comparison of formulae (3.9), (3.12), and (5.3) shows that each of the four terms in expression (3.9) contributes to the numerator $P(t)$ a term of degree $2 d+2 n-4$. For some values of $\varkappa$ the polynomials $P(t)$ and $Q(t)$ may have common roots with respect to the variable $t$, hence the number $2 d+2 n-4$ provides the upper bound for the degree of $P(t)$.

As a consequence we have the following upper bound on the degree of the Arctic curve in the generic 'root-of-unity' case.

Corollary 1. In the case of $\alpha=n / d$ where $n$ and $d$ are co-prime integers and $\lambda$ is generic, the function $\Upsilon(x, y ; \lambda)$ in (2.4) is a polynomial in $x$ and $y$ of total degree less than or equal to $4(n+d)-10$.

Let us now address a more refined question about the degree of $P(t)$ in the special case of $d=2$ and $n$ odd (i.e., when $\alpha$ is half-integer) at $\lambda=\pi / 2$. As we have seen in our examples above, the degree drops down, in 
comparison with the case of generic $\lambda$. We also observed that no such drop down of the degree occurs, e.g., in the cases of integer values of $\alpha$.

Here we have the following result.

Proposition 2. If $\alpha=n / 2$ where $n$ is odd, and $\lambda=\pi / 2$, then

$$
\operatorname{deg} P(t)=2 n-4 \text {. }
$$

PROOF. We start with noting that for arbitrary $\alpha=d / n$ the value $\lambda=\pi / 2$ corresponds to $\varkappa=\pi / 4 \alpha$ and hence we have

$$
\frac{\varkappa}{d}=\frac{\pi}{4 n} \text {. }
$$

Let us now set $d=2$ and consider $n$ odd. In addition to the relations $v_{0}=w_{0}$ and $u_{1}=w_{n-1}$ which are just relations (5.1), in the presently considered case we have, in virtue of (5.5), the relations

$$
v_{1}=-w_{\frac{n-1}{2}}, \quad u_{0}=-w_{\frac{n+1}{2}} .
$$

Due to these two additional relations we see that the common denominator $Q(t)$ in (3.12) coincides with the denominator of the last term in (3.9), that is

$$
Q(t)=\prod_{j=0}^{n-1}\left(t-w_{j}\right)\left(t+w_{j}\right)
$$

Recalling that $d=2$ and inspecting the degrees of the numerators we thus arrive at (5.4).

Therefore we have obtained the following statement.

Corollary 2. If $\alpha=n / 2$ where $n$ is odd, and $\lambda=\pi / 2$, then the Arctic curve is given by an equation in $x$ and $y$ of degree less than or equal to $4 n-10$.

Note that here we cannot claim exact equality, conversely to formula (5.4). As a matter of fact, the exact degree of the curve can hardly be controlled, since it is difficult to find in general the number of components of the algebraic curve resulting from the condition of vanishing discriminant of $P(t)$. Indeed, as we have learned from the example of $\alpha=5 / 2$, the occurrence of more than one component immediately implies that the Arctic curve has lower degree with respect to the one implied by the degree of $P(t)$. An interesting open question concerns therefore the possibility of providing some classification for the irreducible algebraic curves associated to the expression $\Upsilon(x, y ; \lambda)$ for the Arctic curve.

\section{Acknowledgments}

The first author (FC) acknowledges partial support from MIUR, PRIN grant 2007JHLPEZ, and from the European Science Foundation program INSTANS. The third author (AGP) was supported by the Alexander von Humboldt Foundation. AGP also acknowledges partial support from INFN, Sezione di Firenze, from the Russian Foundation for Basic Research (grant 
10-01-00600), and from the Russian Academy of Sciences program "Mathematical Methods in Nonlinear Dynamics".

\section{Appendix A. Discriminant of a polynomial}

Let $P(t)$ be a polynomial of degree $m(m \geqslant 2)$ with non-vanishing leading coefficient, $p_{m} \neq 0$, and let $r_{i}(i=1, \ldots, m)$ be the roots of this polynomial,

$$
P(t)=\sum_{k=0}^{m} p_{k} t^{k}=p_{m} \prod_{i=1}^{m}\left(t-r_{i}\right) .
$$

The discriminant of polynomial $P(t)$, denoted as $D_{m}(P)$, where the subscript refers to the degree of the polynomial, is defined as (sometimes another definition is used, differing by an overall factor $(-1)^{m(m-1) / 2}$ cf. [22, 23]):

$$
D_{m}(P):=p_{m}^{2 m-2} \prod_{i<k}\left(r_{i}-r_{k}\right)^{2} .
$$

It is clear that the condition that $P(t)$ has a double root is equivalent to:

$$
D_{m}(P)=0 \text {. }
$$

We now explain how an explicit expression can be obtained for $D_{m}(P)$, from the knowledge of the coefficients (rather than of the roots) of polynomial $P(t)$. For this aim it is useful to first rephrase equation (A.3) as the condition that two polynomials, say $P(t)$ and $P^{\prime}(t)$ (see below for a slightly different choice) have a common root.

It is well known (see, e.g., 22, 23]) that two arbitrary polynomials have a common (in general, complex) root if and only if the determinant of the Sylvester matrix, i.e. the resultant, of these polynomials vanishes. To be more specific, let $A(t)$ and $B(t)$ be polynomials in $t$, of degrees $m_{A}$ and $m_{B}$ respectively $\left(a_{m_{A}} \neq 0\right.$ and $\left.b_{m_{B}} \neq 0\right)$,

$$
A(t)=\sum_{k=0}^{m_{A}} a_{k} t^{k}, \quad B(t)=\sum_{k=0}^{m_{B}} b_{k} t^{k} .
$$

The Sylvester matrix associated with the polynomials $A(t)$ and $B(t)$ is an $\left(m_{A}+m_{B}\right)$-by- $\left(m_{A}+m_{B}\right)$ matrix, denoted $S_{m_{A}, m_{B}}(A, B)$, where the subscripts refers to the degrees of $A(t)$ and $B(t)$, which is given by the formula:

$$
S_{m_{A}, m_{B}}(A, B)=\left(\begin{array}{cccccccc}
a_{0} & a_{1} & \cdots & a_{m_{A}-1} & a_{m_{A}} & 0 & \ldots & 0 \\
0 & a_{0} & a_{1} & \ldots & a_{m_{A}-1} & a_{m_{A}} & \ldots & 0 \\
\vdots & \ddots & \ddots & \ddots & \ddots & \ddots & \ddots & \vdots \\
0 & \ldots & 0 & a_{0} & a_{1} & \ldots & a_{m_{A}-1} & a_{m_{A}} \\
b_{0} & b_{1} & \ldots & b_{m_{B}-1} & b_{m_{B}} & 0 & \ldots & 0 \\
0 & b_{0} & b_{1} & \ldots & b_{m_{B}-1} & b_{m_{B}} & \ldots & 0 \\
\vdots & \ddots & \ddots & \ddots & \ddots & \ddots & \ddots & \vdots \\
0 & \ldots & 0 & b_{0} & b_{1} & \ldots & b_{m_{B}-1} & b_{m_{B}}
\end{array}\right) .
$$


The requirement that polynomials $A(t)$ and $B(t)$ have a common root is equivalent to the condition

$$
\operatorname{det} S_{m_{A}, m_{B}}(A, B)=0 .
$$

The complete proof of this statement can be found e.g. in [22,23]. Here we shall just show that condition (A.6) is necessary. Indeed, let $\vec{\tau}_{m}$ denote an $m$-component column vector with entries $\left(\vec{\tau}_{m}\right)_{i}=t^{i-1}(i=1, \ldots, m)$ then

$$
S_{m_{A}, m_{B}}(A, B) \vec{\tau}_{m_{A}+m_{B}}=\left(\begin{array}{l}
A(t) \vec{\tau}_{m_{B}} \\
B(t) \vec{\tau}_{m_{A}}
\end{array}\right)=0 .
$$

Thus, if $A(t)$ and $B(t)$ simultaneously vanish for some value of $t$, then the Sylvester matrix has at least one vanishing eigenvalue and hence formula (A.6) follows.

Given polynomial $P(t)$, the standard way of obtaining its discriminant (which is sometimes used as a definition of $D_{m}(P)$, instead of (A.2)) is:

$$
D_{m}(P)=\frac{(-1)^{m(m-1) / 2}}{p_{m}} \operatorname{det} S_{m, m-1}\left(P, P^{\prime}\right) .
$$

Taking into account that

$$
P^{\prime}(t)=m p_{m} t^{m-1}+(m-1) p_{m-1} t^{m-2}+\cdots+2 p_{2} t+p_{1},
$$

let us consider polynomial $\tilde{P}(t)=m P(t)-t P^{\prime}(t)$, which reads:

$$
\tilde{P}(t)=p_{m-1} t^{m-1}+2 p_{m-2} t^{m-2}+\cdots+(m-1) p_{1} t+m p_{0} .
$$

Noting that the system of equations $P(t)=0, P^{\prime}(t)=0$ can be replaced by the system of equations $\tilde{P}(t)=0, P^{\prime}(t)=0$, we can therefore obtain equation (A.3) using the Sylvester determinant associated with the polynomials $\tilde{P}(t)$ and $P^{\prime}(t)$. Furthermore, this can also be extended to the expression for $D_{m}(P)$ regardless of the requirement of its vanishing. Indeed, using the standard properties of determinants, formula (A.8) can be simplified to

$$
D_{m}(P)=(-1)^{m(m-1) / 2} \operatorname{det} S_{m-1, m-1}\left(\tilde{P}, P^{\prime}\right),
$$

which is exactly formula (3.13).

\section{References}

[1] H. Cohn, N. Elkies, and J. Propp, Local statistics for random domino tilings of the Aztec diamond, Duke Math. J. 85 (1996), 117-166, arXiv math/0008243.

[2] W. Jockush, J. Propp, and P. Shor, Random domino tilings and the arctic circle theorem, arXiv math/9801068.

[3] H. Cohn, M. Larsen, and J. Propp, The shape of a typical boxed plane partition, New York J. Math. 4 (1998), 137-165, arXiv math/9801059

[4] A. Borodin, V. Gorin, and E. M. Rains, q-Distributions on boxed plane partitions, Selecta Math. (N.S.) 16 (2010), no. 4, 731-789, arXiv:0905.0679.

[5] V. E. Korepin, Calculations of norms of Bethe wave functions, Commun. Math. Phys. 86 (1982), 391-418.

[6] A. G. Izergin, Partition function of the six-vertex model in the finite volume, Sov. Phys. Dokl. 32 (1987), 878-879. 
[7] A. G. Izergin, D. A. Coker, and V. E. Korepin, Determinant formula for the six-vertex model, J. Phys. A 25 (1992), 4315-4334.

[8] K. Eloranta, Diamond ice, J. Stat. Phys. 96 (1999), 1091-1109.

[9] V. E. Korepin and P. Zinn-Justin, Thermodynamic limit of the six-vertex model with domain wall boundary conditions, J. Phys. A 33 (2000), 7053-7066, arXiv: cond-mat/0004250

[10] P. Zinn-Justin, Six-vertex model with domain wall boundary conditions and onematrix model, Phys. Rev. E 62 (2000), 3411-3418, arXiv math-ph/0005008

[11] P. Zinn-Justin, The influence of boundary conditions in the six-vertex model, arXiv: cond-mat/0205192

[12] O. F. Syljuåsen and M. B. Zvonarev, Monte-Carlo simulations of vertex models, Phys. Rev. E 70 (2004), 016118, arXiv cond-mat/0401491

[13] D. Allison and N. Reshetikhin, Numerical study of the 6-vertex model with domain wall boundary conditions, Ann. Inst. Fourier (Grenoble) $\mathbf{5 5}$ (2005), 1847-1869, arXiv: cond-mat/0502314

[14] N. Reshetikhin, Lectures on the integrability of the 6-vertex model, arXiv 1010.5031.

[15] F. Colomo and A. G. Pronko, The limit shape of large alternating-sign matrices, SIAM J. Discrete Math. 24 (2010), no. 4, 1558-1571, arXiv 0803.2697.

[16] F. Colomo and A. G. Pronko, The arctic curve of the domain-wall six-vertex model, J. Stat. Phys. 138 (2010), no. 4, 662-700, arXiv 0907.1264

[17] F. Colomo, A. G. Pronko, and P. Zinn-Justin, The arctic curve of the domain-wall six-vertex model in its anti-ferroelectric regime, J. Stat. Mech. (2010), L03002, arXiv: 1001.2189 .

[18] R. Kenyon, A. Okounkov, and S. Sheffield, Dimers and amoebae, Ann. of Math. 163 (2006), 1019-1056, arXiv math-ph/0311005

[19] R. Kenyon and A. Okounkov, Planar dimers and Harnack curves, Duke Math. J. 131 (2006), 499-523, arXiv math.AG/0311062.

[20] R. Kenyon and A. Okounkov, Limit shapes and the complex Burgers equation, Acta Math. 199 (2007), 263-302, arXiv math-ph/0507007.

[21] F. Colomo and A. G. Pronko, The Arctic Circle revisited, Contemp. Math. 458 (2008), 361-376, arXiv 0704.0362.

[22] D. Cox, J. Little, and D. O'Shea, Ideals, varieties and algorithms, 3rd ed., Springer, 2007.

[23] I. M. Gelfand, M. M. Kapranov, and A. V. Zelevinsky, Discriminants, resultants and multidimensional determinants, Mathematics: Theory and Applications, Birkhauser, 1994.

InfN, Sezione di Firenze, Via G. Sansone 1, 50019 Sesto Fiorentino (Fi), ITALY

E-mail address: colomo@fi.infn.it

Department of Mathematics, University of Pisa, Largo Bruno Pontecorvo 5, 56127 Pisa, ItAly

E-mail address: noferini@mail.dm.unipi.it

Department of Physics, University of Wuppertal, 42097 Wuppertal, Germany (On leave of absence from: Saint Petersburg Department of V. A. Steklov Mathematical Institute, Russian Academy of Sciences, Fontanka 27, 191023 Saint Petersburg, Russia)

E-mail address: agp@pdmi.ras.ru 